

\title{
First Principles Studies on One-Photon and Two-Photon Absorption Properties of Symmetric Thiophene Derivatives
}

Chungang $\mathrm{Li}^{*}$ and Zhinong WAng

Longkou Entry-exit Inspection and Quarantine Bureau, Longkou 265700, Shandong, P.R. China

*Corresponding author: E-mail: lichungang163@163.com

Received: 12 July 2013;

Accepted: 21 November 2013;

Published online: 23 June 2014;

AJC-15379

In this paper, the equilibrium geometries, one-photon, two-photon absorption properties and the transition nature of three thiophene derivatives have been theoretically studied by applying density functional response theory. Our calculated results have confirmed that the one-photon and two-photon absorption are enhanced and bathochromically shift with the increase of the strength of the D end-groups as well as the length of the conjugated system. The nonlinear optical phenomenons of the derivatives under investigation, originating from the intramolecular charge transfer within the $\pi$-conjugated system.

Keywords: One-photon absorption, Two-photon absorption, Density functional theory, Thiophene derivatives.

\section{INTRODUCTION}

Multi-photon absorption is a nonlinear optical process where a molecule absorb two or more photons, simultaneously, exciting from ground state to an excited state. Multi-photon absorption is currently the focus of intensive research efforts because of their potential civilian and military applications in the fields of multi-photon fluorescence imaging, 3D optical data storage, optical limiting, photodynamic therapy and microfabrication. Numerous investigations have been taken on organic materials both experimentally and theoretically, showing that, in general, increasing the planarity, prolonging the $\pi$-bridge length and strengthening the donor and/or acceptor ability, will enhance the nonlinear optical effect ${ }^{1-6}$.

A stream of conjugated organic units with a structure of $D(A)-\pi-D(A)$ and $D(A)-\pi-D(A)-\pi-D(A)$ attracted increasing attention, having been designed and synthesized as novel multiphoton absorption materials where $\mathrm{D}$ and $\mathrm{A}$ denote electron donating and accepting groups, such as triphenylamine ${ }^{7,8}$, fluorene ${ }^{9-11}$, etc. Amongst them, donor-acceptor (D-A) type conjugated system have been paid much attention as efficient nonlinear optical materials, which has been regarded as an effective method to reduce the band gap ${ }^{12}$. Thiophenes and their derivatives are of special interest among the D-A type conjugated systems, experimental and theoretical studies revealed that such compounds are strong candidates for potential use in non-linear optical materials ${ }^{13}$. Studies show that thiophene derivatives are versatile building blocks for the synthesis of donor-acceptor substituted $\pi$-conjugated systems for several optical applications, however, only scarce examples of (oligo-) thiophene derivatives have been investigated for two-photon ${ }^{14,15}$. Investigations indicate that the introduction of proper electron donating and withdrawing entities can enhance the optical nonlinearity of such compounds.

Keeping this in view, we report the theoretical study of three thiophene derivatives with different end capped groups, methyl, ethyl and phenyl, whose structures are sketched in Fig. 1. A state-of-the-art computational method which applies first principles calculations has been employed to study the one-photon and two-photon absorption properties of the compounds. The comparison among the three thiophene derivatives will be discussed in details. Moreover, we will try to highlight different response behaviors between different processes. Here we will challenge to present: (1) systematic studies on one-photon absorption and two-photon absorption; (2) discussions on the different optical responses between different nonlinear optical; (3) a detailed comparison of theoretical one-photon absorption and two-photon absorption properties between the compounds.

\section{COMPUTATIONAL DETAILS}

All geometries of the three derivatives investigated were optimized at Becke's three-parameter Lee-Yang-Parr (B3LYP) level, in gas phase, by employing the Gaussian 09 program $^{19}$. The properties calculations of both one-photon absorption and two-photon absorption of the derivatives were performed at 




(a)

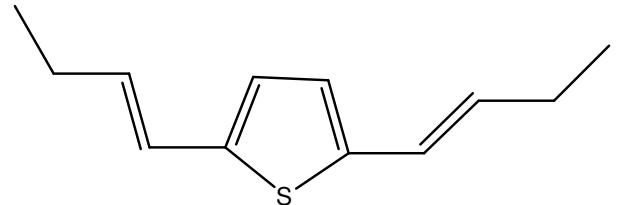

(b)

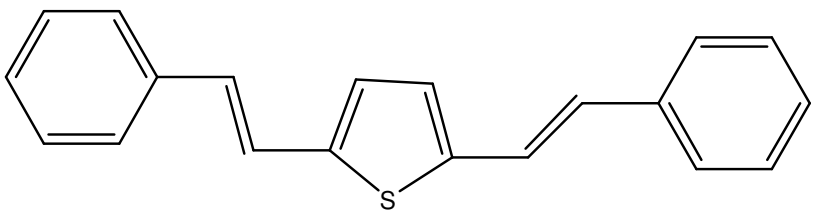

(c)

Fig. 1. Molecular structure of the three thiophene derivatives with different end capped groups, methyl, ethyl, and phenyl

B3LYP level. In our calculation, the properties of one-photon absorption were obtained from a normal TDDFT calculation by Gaussian 09, while two-photon absorption has been done by using the parallel implementation of the quadratic ${ }^{20}$ response module implemented in Dalton ${ }^{21}$. All the calculations is performed with 6-31++G(d,p) basis set, including the geometries and properties calculations of all the compounds under investigation.

One-photon absorption: For one-photon absorption (OPA), the extinction coefficient can be written as ${ }^{16,17}$,

$$
\varepsilon_{\mathrm{OPA}}(\omega)=\frac{(2 \pi)^{2} \times \omega \times \mathrm{N}_{\mathrm{A}}}{3 \times 1000 \times \ln (10) \times \hbar \mathrm{c}_{0} \times\left(4 \pi \varepsilon_{0}\right)} \sum_{\alpha=\mathrm{x}, \mathrm{y}, \mathrm{z}}\left|\mu_{\mathrm{gf}}^{\alpha}\right|^{2}
$$

where $\mathrm{N}_{\mathrm{A}}=$ Avogadro's number, $\mathrm{c}_{0}=$ speed of light in vacuo and $\varepsilon_{0}=$ vacuum permittivity $\omega=$ circular frequency of the laser. The Cartesian $(\alpha=\mathrm{x}, \mathrm{y}, \mathrm{z})$ component of the transition electric dipole moment from the initial state $|\mathrm{g}\rangle$ to the final state $|\mathrm{f}\rangle$ is evaluated explicitly as

$$
\mu_{\mathrm{gf}}^{\alpha}=\left\langle\mathrm{g}\left|\mu^{\alpha}\right| \mathrm{f}\right\rangle
$$

The last row of eqn. (1) yields the one-photon absorption observable in unit of $\mathrm{L} \mathrm{mol}^{-1} \mathrm{~cm}^{-1}$ starting from circular frequencies and transition dipole moments given in atomic units.

In present paper, we also report the oscillator strength defined as:

$$
\delta_{\mathrm{OPA}}=\frac{2 \omega_{\mathrm{gf}}}{3} \sum_{\alpha}\left|\mu_{\mathrm{gf}}^{\alpha}\right|^{2}
$$

Two-photon absorption (TPA): The two-photon absorption cross section $\sigma_{\mathrm{TPA}}\left(\right.$ for $\left.\omega_{1}, \omega_{2}=\omega\right)$, is given by the expression $^{16}$

$$
\sigma_{\mathrm{TPA}}=\frac{4 \pi^{3} \mathrm{a}_{0}^{5} \alpha}{15 \mathrm{c}_{0}} \omega^{2} \sum_{\mathrm{f}} \mathrm{g}\left(2 \omega, \omega_{\mathrm{gf}}\right) \times \delta_{\mathrm{gf}}^{\mathrm{TPA}}(\omega)
$$

Here $\alpha$ is the fine structure constant, $\alpha_{0}$ Bohr radius and the orientationally averaged two-photon probability $\delta_{\mathrm{gf}}^{\mathrm{TPA}}(\omega)$ is given by ${ }^{18}$

$$
\delta_{\mathrm{gf}}^{\mathrm{TPA}}(\omega)=\sum_{\alpha \beta}\left(F \times S_{\mathrm{gf}}^{\alpha \alpha} S_{\mathrm{gf}}^{\beta \beta, *}+\mathrm{G} \times S_{\mathrm{gf}}^{\alpha \beta} S_{\mathrm{gf}}^{\alpha \beta, *}+\mathrm{H} \times S_{\mathrm{gf}}^{\alpha \beta} S_{\mathrm{gf}}^{\beta \alpha, *}\right)
$$

where $S_{\mathrm{gf}}^{\alpha \beta}$ is the two-photon transition matrix element, which is defined by the sum-over-state (SOS) expression as

$$
S_{\mathrm{gf}}^{\alpha \beta}(\omega)=\frac{1}{\hbar} \sum_{\mathrm{k}}\left[\frac{\left\langle\mathrm{g}\left|\mu_{\alpha}\right| \mathrm{k}\right\rangle\left\langle\mathrm{k}\left|\mu_{\beta}\right| \mathrm{f}\right\rangle}{\omega_{\mathrm{k}}-\omega}+\frac{\left\langle\mathrm{g}\left|\mu_{\beta}\right| \mathrm{k}\right\rangle\left\langle\mathrm{k}\left|\mu_{\alpha}\right| \mathrm{f}\right\rangle}{\omega_{\mathrm{k}}-\omega}\right]
$$

F, $G$ and $H$ assume values of 2, 2, 2 for linearly and -2, 3, 3 for circularly polarized beams, respectively.

The resulting unit of $\sigma_{\mathrm{TPA}}$ is $\mathrm{cm}^{4} \mathrm{~s}$ photon ${ }^{-1}$ molecule ${ }^{-1}$ $\left(10^{-50} \mathrm{~cm}^{4} \mathrm{~s}_{\text {photon }}{ }^{-1}\right.$ molecule ${ }^{-1}=1$ Göppert-Mayer, $\left.1 \mathrm{GM}\right)$ starting from $\mathrm{a}_{0}$ and $\mathrm{c}_{0}$ given in CGS units and circular frequencies and two-photon transition probabilities given in atomic units.

\section{RESULTS AND DISCUSSION}

The one-photon absorption spectra of the three thiophene derivatives calculated with the density functional-B3LYP in gas phase are compared in Fig. 2. The Lorenztian lifetime broadening is set to $0.10 \mathrm{eV}$. Table- 1 collects the excitation energies $\mathrm{E}_{\mathrm{gf}}$ (both in $\mathrm{eV}$ and $\mathrm{nm}$ ), one-photon absorption oscillator strengths $\delta_{\mathrm{OPA}}$ and the corresponding transition natures of the system.

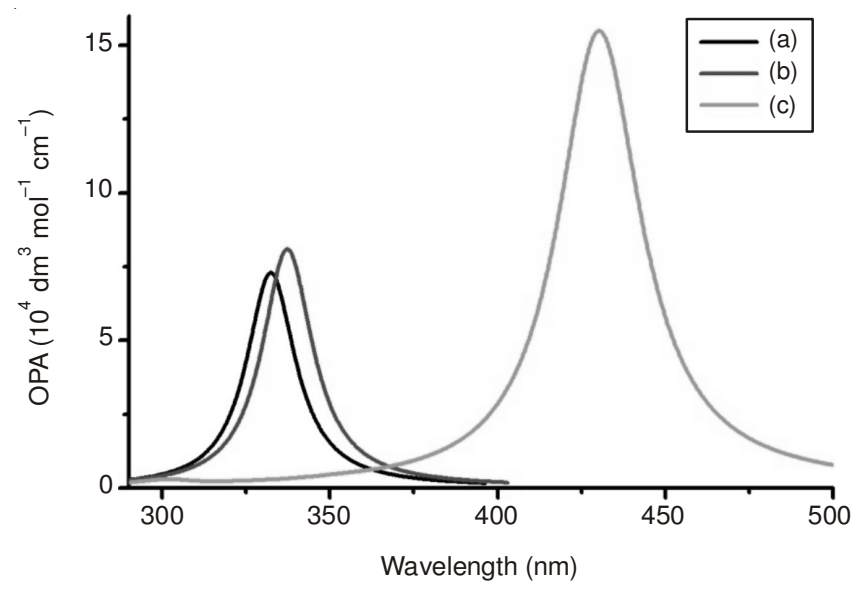

Fig. 2. Calculated one-photon absorption spectra for the three derivatives in gas phase obtained at B3LYP/6-31++G(d,p) level, by summing over the first 6 excited states, convoluted with a Lorentzian broadening lifetime of $0.1 \mathrm{eV}$

Fig. 2 showed that all the three derivatives are predicted with one evident absorption peak, with taking 6 excited states into consideration. The spectra as well as the wavelength of the absorption peak are red-shifted, when the end capped groups vary from methyl to ethyl, to phenyl. The strength of one-photon absorption is in the order of phenyl > ethyl > methyl, as clearly indicated in Fig. 2. That is clear, since the increase of the length of the conjugated system as well as the strength of the D end-groups allows to increase the ability of charge-transfer ${ }^{22}$.

By looking into Table-1, we can inspect into the details under the broadened spectra. It is easy to find that the first (S1) excited state is the strongest one-photon absorption state 
of all the molecules investigated in this system, with the largest one-photon absorption oscillator strength of $0.7975,0.8858$, 1.6935 respectively, for compounds $\mathrm{a}, \mathrm{b}$ and c. The electron transition is originated from the highest occupied molecular orbital (HOMO) to the lowest unoccupied molecular orbital (LUMO), though the derivatives are designed with different end capped groups (Table-1). For example, for methyl, the main excited state $\mathrm{S} 1$ originates from HOMO to LOMO, involving charge transfer within the $\pi$-conjugated system, as show in Fig. 3.

Two-photon absorption: The explicit use of sum-overstate expression [eqn. (6)] is the direct way of modeling a two-photon absorption spectrum, in which all intermediate states should be included. By solving a set of linear matrix equations, the two-photon absorption cross section can be implicitly got within the response theory. ${ }^{23}$ The calculated twophoton absorption cross sections at B3LYP/6-31++G(d,p) level, obtained assuming linearly and circularly polarized laser beam, respectively, are collected in Table-2. The cross sections are for the first 6 excited states and corresponding to the maximum value with Lorenztian lifetime broadening set to $0.10 \mathrm{eV}$, in conformity to the assumptions for one-photon absorption in present study. The corresponding two-photon absorption spectra of the derivatives are plotted in Fig. 4.

\begin{tabular}{|c|c|c|c|c|c|c|}
\hline \multicolumn{7}{|c|}{$\begin{array}{l}\text { TABLE- } 1 \\
\text { EXCITATION ENERGIES } E_{\mathrm{gf}}(\mathrm{eV}) \text {, CORRESPONDING WAVELENGTH } \lambda_{\mathrm{gf}}(\mathrm{NM}), \text { OSCILLATOR STRENGTHS } \delta_{\mathrm{OPA}} \text {, AND } \\
\text { DOMINATING TRANSITION NATURE FOR THE MAIN EXCITED STATES OF COMPOUNDS A, B } \\
\text { AND C IN GAS PHASE, OBTAINED AT B3LYP/6-31++G** LEVEL }\end{array}$} \\
\hline Compound & State & $\mathrm{E}_{\mathrm{gf}}$ & $\lambda_{\mathrm{gf}}$ & $\delta_{\mathrm{OPA}}$ & Transition & Coefficient \\
\hline \multirow[b]{2}{*}{$\mathrm{a}$} & $\mathrm{S}_{1}$ & 3.7294 & 332.45 & 0.7975 & $\mathrm{HOMO} \rightarrow$ LUMO & 0.70480 \\
\hline & $\mathrm{S}_{2}$ & 4.4492 & 278.66 & 0.0007 & $\mathrm{HOMO} \rightarrow \mathrm{LUMO}+1$ & 0.62484 \\
\hline \multirow{2}{*}{ b } & S1 & 3.6753 & 337.35 & 0.8858 & $\mathrm{HOMO} \rightarrow \mathrm{LUMO}$ & 0.70409 \\
\hline & S2 & 4.4076 & 281.30 & 0.0013 & $\mathrm{HOMO} \rightarrow \mathrm{LUMO}+1$ & 0.63178 \\
\hline \multirow{2}{*}{$\mathrm{c}$} & S1 & 2.8803 & 430.46 & 1.6935 & $\mathrm{HOMO} \rightarrow$ LUMO & 0.70820 \\
\hline & S2 & 3.5167 & 352.55 & 0.0032 & $\mathrm{HOMO} \rightarrow \mathrm{LUMO}+1$ & 0.61596 \\
\hline
\end{tabular}
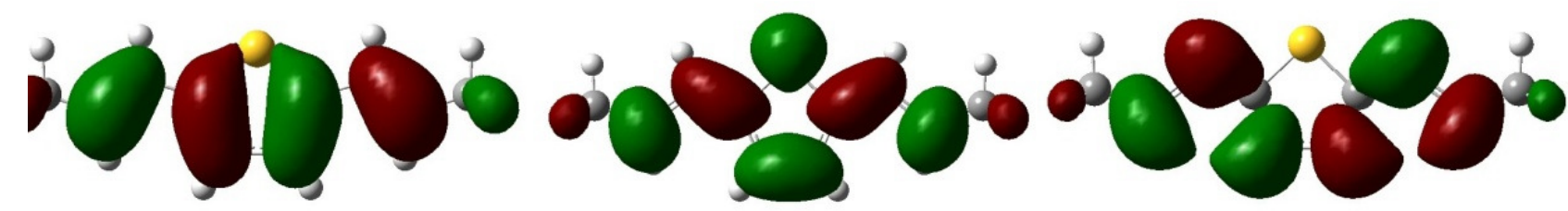

HOMO (a)

LOMO (a)

$\mathrm{LOMO}+1$ (a)
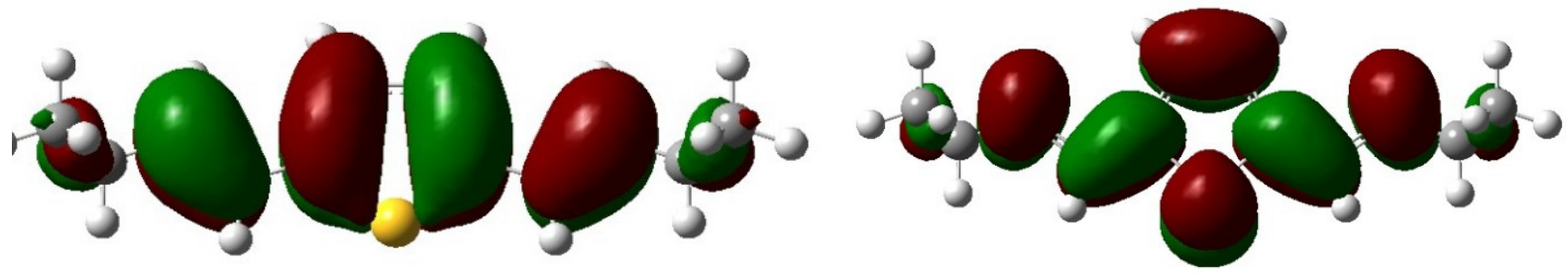

HOMO (b)

LOMO (b)

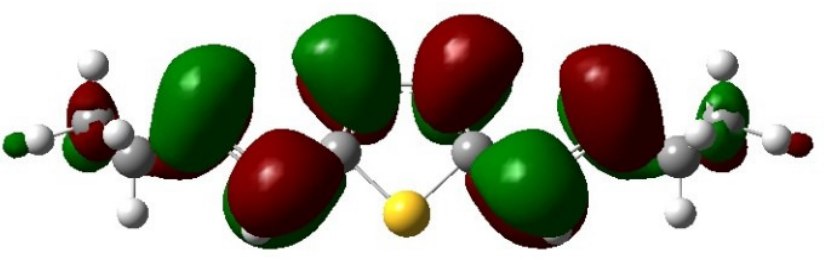

$\mathrm{LOMO}+1(\mathrm{~b})$
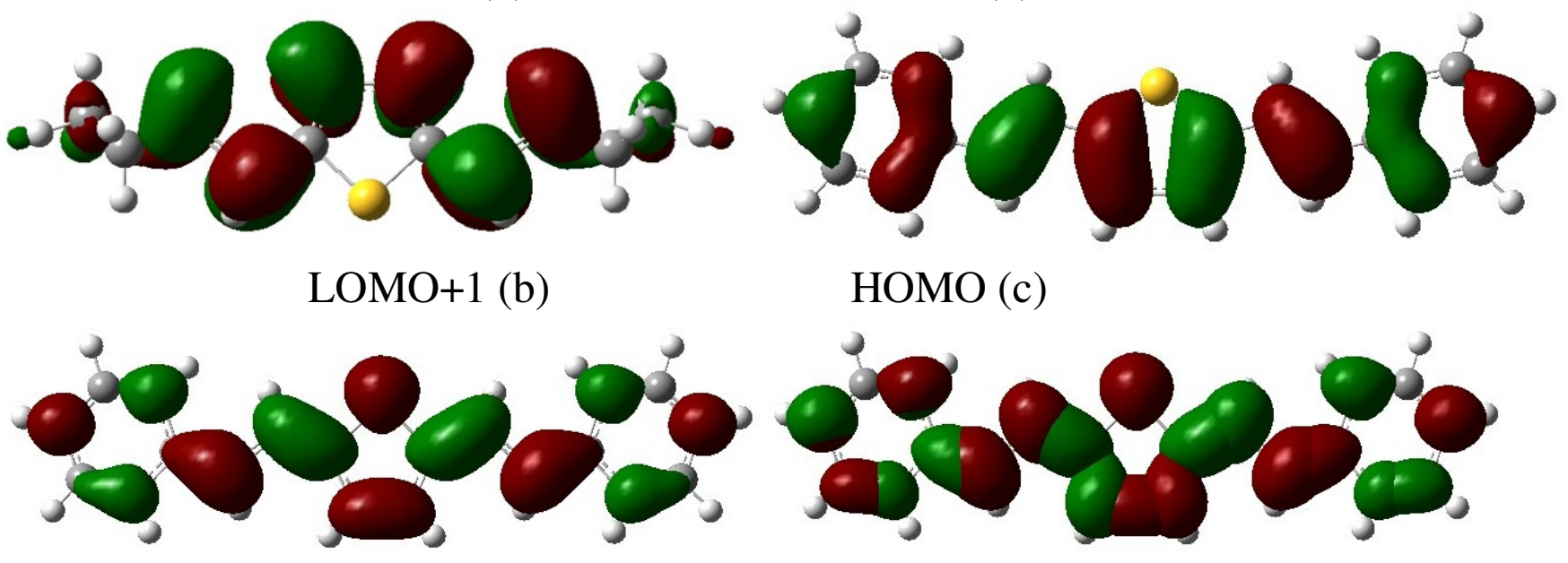

HOMO (c)

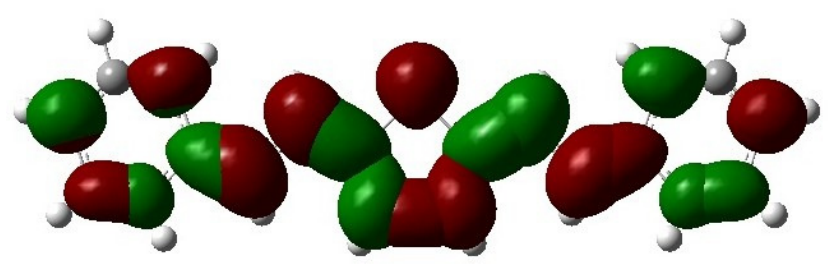

LOMO (c)

$\mathrm{LOMO}+1(\mathrm{c})$

Fig. 3. Molecular orbitals for compounds a, b, and c optimized at B3LYP/6-31++G(d,p) level in gas phase 
TABLE-2

CALCULATED TWO-PHOTON ABSORPTION CROSS SECTIONS FOR THE FIRST 6 LOWEST EXCITED STATES OF COMPOUNDS a, b AND c IN GAS PHASE, OBTAINED BY USING RESPECTIVELY LINEARLY AND CIRCULARLY POLARIZED LASER BEAM, AT B3LYP/6-31++G**LEVEL. LORENTZIAN LIFETIME BROADENING OF $0.1 \mathrm{eV}$

\begin{tabular}{|c|c|c|c|c|c|c|}
\hline \multirow{2}{*}{ State } & \multicolumn{2}{|c|}{ Compound a } & \multicolumn{2}{|c|}{ Compound b } & \multicolumn{2}{|c|}{ Compound c } \\
\hline & $\sigma_{\mathrm{TPA}}^{\operatorname{lin}}$ & $\sigma_{\mathrm{TPA}}^{\mathrm{cir}}$ & $\sigma_{\mathrm{TPA}}^{\text {lin }}$ & $\sigma_{\mathrm{TPA}}^{\mathrm{cir}}$ & $\sigma_{\mathrm{TPA}}^{\operatorname{lin}}$ & $\sigma_{\mathrm{TPA}}^{\mathrm{cir}}$ \\
\hline S1 & 0.03 & 0.04 & 0.06 & 0.10 & 0.43 & 0.64 \\
\hline S3 & 0.02 & 0.03 & 0.18 & 0.74 & 362.51 & 245.21 \\
\hline S4 & 0.11 & 0.16 & 3.72 & 5.59 & 14.19 & 21.28 \\
\hline S5 & 0.01 & 0.02 & 52.45 & 27.37 & 5.60 & 3.70 \\
\hline
\end{tabular}



Fig. 4. Calculated two-photon absorption spectra for the thiophene derivatives in gas phase obtained at B3LYP/6-31++G(d,p) level, taking the first 6 excited states into consideration, convoluted with a Lorentzian broadening lifetime of $0.1 \mathrm{eV}$

Compound a gives a strong absorption in the range of 250-300 nm, however, for ethyl and phenyl, two absorption peaks can be seen in the wavelength of 250-300 and 250-400 nm, respectively. It should be stressed that there might be other large absorption bands above the predicted wavelength, corresponding to electronic states higher than 6 , out of the present range under investigation. The spectra indicate that two-photon absorption is much red-shifted and increased for phenyl, with respect to methyl and ethyl. This can be understood due to the fact that ethyl has stronger electron-donor strength than methyl, which can build up stronger charge transfer and possess larger two-photon absorption cross section. It is worth to mention that the conjugation of the system also play an important role in enhancing two-photon absorption, since stwo-photon absorption of compound c (end-group of phenyl) is much larger than the other two derivatives.

One is easy methods to find that the second excited state $\left(\mathrm{S}_{2}\right)$ associated with the combined transition from $\mathrm{HOMO} \rightarrow$ $\mathrm{LUMO}+1$ is the dominant contribution to the major absorption peak for the three derivatives (Table-2). The corresponding charge transfer nature can be seen from Fig. 3. It can be noticed that the two-photon absorption cross section for phenyl is the largest one in the derivatives under investigation. Taking the values for the linearly polarized lights as an example, the maximum two-photon absorption cross section of phenyl (1678 $\mathrm{GM})$ is 5.8 times of that for ethyl (288 GM) and 10.8 times of that for methyl (156 GM). However, the strongest one-photon absorption state $S_{1}$ have very weak two-photon absorption intensity, with $\sigma_{\mathrm{TP}}^{\text {lin }}$ of only $0.03,0.06$ and $0.43 \mathrm{GM}$ for methyl, ethyl and phenyl, respectively due to different selection rules.

For the state responsible for the highest peak, i.e. $\mathrm{S}_{1}$, the two-photon absorption cross sections for linearly polarized light are larger than those for the circularly polarized light. For instance, the ratio of $\sigma_{\mathrm{TP}}^{\mathrm{lin}} / \sigma_{\mathrm{TP}}^{\mathrm{cir}}$ is around 1.4 for methyl, which can be understood since for this state, $S_{x x}$ dominate the two-photon absorption cross section. However, not for all the states investigated share the same rule. For example, $S_{1}$ for methyl, is larger than the corresponding $\sigma_{\mathrm{TPA}}^{\text {lin }}$, since for this state, the two-photon absorption cross section is mostly determined by the two-photon transition matrix component $\mathrm{S}_{\mathrm{xy}}\left(\right.$ and $\left.\mathrm{S}_{\mathrm{yx}}\right)$.

\section{Conclusion}

In this contribution the one-photon and two-photon absorption properties of three thiophene derivatives have been investigated. Our calculations have confirmed that the increase of the electron-donor strength as well as the conjugation will benefit the one-photon and two-photon absorption. Such enhancement of the one-photon absorption and two-photon absorption responses is associated with a predominant intramolecular charge-transfer character within the $\pi$-conjugated system, larger charge transfer within the molecules will certainly lead to enhanced nonlinear optical responses. In addition, increasing the electron-donor strength as well as the conjugation will lead to a significant bathochromic shift of both one-photon and two-photon absorption in present system. The three thiophene derivatives under investigation in this present system are all promising nonlinear optical materials.

\section{REFERENCES}

1. B. Reinhardt, Photonics Sci. News, 4, 21 (1998).

2. M. Pawlicki, H.A. Collins, R.G. Denning and H.L. Anderson, Angew. Chem. Int. Ed., 48, 3244 (2009).

3. K.D. Belfield, D.J. Hagan, E.W. Van Stryland, K.J. Schafer and R.A. Negres, Org. Lett., 1, 1575 (1999).

4. S.-J. Chung, T.-C. Lin, K.-S. Kim, G.S. He, J. Swiatkiewicz, P.N. Prasad, G.A. Baker and F.V. Bright, Chem. Mater., 13, 4071 (2001).

5. K.D. Belfield, A. Morales, V. M. Chapela and J. Percino, Polymer Prepr., 44, 1061 (2003).

6. C.C. Corredor, Z.L. Huang, K.D. Belfield, A.R. Morales and M.V. Bondar, Chem. Mater., 19, 5165 (2007).

7. C. Katan, F. Terenziani, O. Mongin, M.H.V. Werts, L. Porrès, T. Pons, J. Mertz, S. Tretiak and M. Blanchard-Desce, J. Phys. Chem. A, 109, 3024 (2005). 
8. G. Ramakrishna and T. Goodson, J. Phys. Chem. A, 111, 993 (2007).

9. A.R. Morales, K.D. Belfield, J.M. Hales, E.W. Van Stryland and D.J. Hagan, Chem. Mater., 18, 4972 (2006).

10. R. Fortrie, R. Anemian, O. Stephan, J.-C. Mulatier, P.L. Baldeck, C. Andraud and H. Chermette, J. Phys. Chem. C, 111, 2270 (2007).

11. J.E. Rogers, J.E. Slagle, D.G. McLean, R.L. Sutherland, M.C. Brant, J. Heinrichs, R. Jakubiak, R. Kannan, L.S. Tan and P.A. Fleitz, J. Phys. Chem. A, 111, 1899 (2007).

12. Y. Zhang, S.K. Hau, H.L. Yip, Y. Sun, O. Acton and A.K.Y. Jen, Chem. Mater., 22, 2696 (2010)

13. C. Herbivo, A. Comel, G. Kirsch, A.M.C. Fonseca, M. Belsley and M.M.M. Raposo, Dyes Pigments, 86, 217 (2010).

14. T. Narita, M. Takase, T. Nishinaga, M. Iyoda, K. Kamada and K. Ohta, Chem. Eur. J., 16, 12108 (2010).

15. S. Ellinger, K.R. Graham, P. Shi, R.T. Farley, T.T. Steckler, R.N. Brookins, P. Taranekar, J. Mei, L.A. Padilha, T.R. Ensley, H. Hu, S. Webster, D.J. Hagan, E.W. Van Stryland, K.S. Schanze and J.R. Reynolds, Chem. Mater., 23, 3805 (2011).

16. D. P. Craig, T. Thirunamachandran, Molecular Quantum Electrodynamics, Academic Press Inc., London (1984).

17. I. Tinoco, J. Chem. Phys., 62, 1006 (1975).

18. F. Helmchen and W. Denk, Curr. Opin. Neurobiol., 12, 593 (2002).
19. M.J. Frisch, G.W. Trucks, H.B. Schlegel, G.E. Scuseria, M.A. Robb, J.R. Cheeseman, G. Scalmani, V. Barone, B. Mennucci, G.A. Petersson, H. Nakatsuji, M. Caricato, X. Li, H.P. Hratchian, A.F. Izmaylov, J. Bloino, G. Zheng, J.L. Sonnenberg, M. Hada, M. Ehara, K. Toyota, R. Fukuda, J. Hasegawa, M. Ishida, T. Nakajima, Y. Honda, O. Kitao, H. Nakai, T. Vreven, J.A. Montgomery Jr., J.E. Peralta, F. Ogliaro, M. Bearpark, J.J. Heyd, E. Brothers, K.N. Kudin, V.N. Staroverov, T. Keith, R. Kobayashi, J. Normand, K. Raghavachari, A. Rendell, J.C. Burant, S.S. Iyengar, J. Tomasi, M. Cossi, N. Rega, J.M. Millam, M. Klene, J.E. Knox, J.B. Cross, V. Bakken, C. Adamo, J. Jaramillo, R. Gomperts, R.E. Stratmann, O. Yazyev, A.J. Austin, R. Cammi, C. Pomelli, J.W. Ochterski, R.L. Martin, K. Morokuma, V.G. Zakrzewski, G.A. Voth, P. Salvador, J.J. Dannenberg, S. Dapprich, A.D. Daniels, O. Farkas, J.B. Foresman, J.V. Ortiz, J. Cioslowski and D.J. Fox, Gaussian 09, Revision A. 02, Gaussian, Inc.: Wallingford, CT (2009).

20. L. Frediani, H. Ågren, L. Ferrighi and K. Ruud, J. Chem. Phys., 123, 144117 (2005).

21. DALTON, A Molecular Electronic Structure Program, Release 2.0; http: //www.kjemi.uio.no/software/dalton/dalton.html (2005).

22. E. Genin, V. Hugues, G. Clermont, C. Herbivo, M.C.R. Castro, A. Comel, M.M.M. Raposo and M. Blanchard-Desce, Photochem. Photobiol., 11, 1756 (2012).

23. J. Olsen and P. Jorgensen, J. Chem. Phys., 82, 3235 (1985). 